\title{
Sphingomonas panaciterrae sp. nov., a plant growth-promoting bacterium isolated from soil of a ginseng field
}

\author{
Johan Sukweenadhi ${ }^{1}$ Yeon-Ju Kim ${ }^{2}$ - Chang Ho Kang ${ }^{3}$ Mohamed El-Agamy Farh' ${ }^{1}$ \\ Ngoc-Lan Nguyen ${ }^{2}$ - Van-An Hoang ${ }^{2}$ Eul-Su Choi ${ }^{4} \cdot$ Deok-Chun Yang ${ }^{1,2}$
}

Received: 13 March 2015 / Revised: 9 June 2015 / Accepted: 28 June 2015

(C) Springer-Verlag Berlin Heidelberg 2015

\begin{abstract}
Strain DCY91 ${ }^{\mathrm{T}}$, a Gram-stain-negative, rodshaped, aerobic, non-motile bacterium, was isolated from soil of ginseng field in Gyeonggi province, South Korea. Strain DCY91 ${ }^{\mathrm{T}}$ shared the highest $16 \mathrm{~S}$ rRNA gene sequence similarity with Sphingomonas mucosissima DSM $17494^{\mathrm{T}}(98.55 \%)$, Sphingomonas dokdonensis KACC $17420^{\mathrm{T}}(98.11 \%)$ and Sphingomonas xinjiangensis DSM $26736^{\mathrm{T}}(96.68 \%)$. The strain $\mathrm{DCY}^{\mathrm{T}}{ }^{\mathrm{T}}$ was found to able to grow best in trypticase soy agar at $28{ }^{\circ} \mathrm{C}$, at $\mathrm{pH} 7$ and at $0.5 \% \mathrm{NaCl}$. Ubiquinone 10 was identified as the isoprenoid quinone. The major polar lipids were identified as sphingoglycolipid, diphosphatidylglycerol, phosphatidylethanolamine, phosphatidylglycerol and phosphatidylcholine. The major fatty acids of strain
\end{abstract}

Communicated by Erko Stackebrandt.

Electronic supplementary material The online version of this article (doi:10.1007/s00203-015-1134-z) contains supplementary material, which is available to authorized users.

Yeon-Ju Kim

yeonjukim@khu.ac.kr

$\bowtie$ Deok-Chun Yang

dcyang@khu.ac.kr

1 Graduate School of Biotechnology and Ginseng Bank, College of Life Science, Kyung Hee University, Yongin 446-701, Korea

2 Department of Oriental Medicinal Biotechnology, College of Life Science, Kyung Hee University, Yongin 446-701, Korea

3 Division of Applied Life Science and PMBBRC, Gyeongsang National University, 501 Jinju-daero, Jinju 660-701, Korea

4 GyeonggiDo Agricultural Research and Extension Services, Hwaseong, Gyeonggi-do, Korea
DCY $91^{\mathrm{T}}$ were identified as unsaturated $\mathrm{C}_{18: 1} \omega 7 c$ and saturated $\mathrm{C}_{16: 0}$. The major polyamine content was sym-homospermidine. The DNA G $+\mathrm{C}$ content was determined to be $65.8 \mathrm{~mol} \%$ (HPLC). After 6 days of incubation, strain DCY $91^{\mathrm{T}}$ produced $9.64 \pm 1.73$ and $33.73 \pm 4.66 \mu \mathrm{g} / \mathrm{ml}$ indole-3-acetic acid, using media without L-tryptophan and supplemented with L-tryptophan, respectively. Strain DCY $91^{\mathrm{T}}$ was also weakly solubilized phosphate and produced siderophores. On the basis of the phenotypic characteristics, genotypic analysis and chemotaxonomic characteristics, strain $\mathrm{DCY} 91^{\mathrm{T}}$ is considered to represent a novel species of the genus Sphingomonas, for which the name Sphingomonas panaciterrae sp. nov. is proposed.

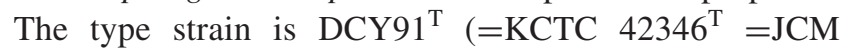
$30807^{\mathrm{T}}$ ).

Keywords Sphingomonas panaciterrae · Ginseng soil IAA producer

\section{Introduction}

The genus Sphingomonas belongs to the Alphaproteobacteria (Lee et al. 2005) and was firstly described by Yabuuchi et al. (1990). The description of the genus was subsequently emended by Takeuchi et al. (2001) with the type strain Sphingomonas paucimobilis. At the time of writing, the genus Sphingomonas comprised 88 species with validly published names as of January 2015 (http://www. bacterio.net/sphingomonas.html). Members of the genus Sphingomonas are rod-shaped, yellow-pigmented, nonsporulating, aerobic, chemoheterotrophic, Gram-stainingnegative and non-motile or motile by means of a single polar flagellum (Busse et al. 2003). They are characterized chemotaxonomically by the presence of ubiquinone 10 